\title{
Fault Feature Extraction for Anchor Bolt Loosening of Escalator Based on EWT and Bispectrum Analysis
}

\author{
Zheng Yan* \\ China Railway Siyuan Survey and Design Group Co., LTD., Wuhan, Hubei, China \\ *Corresponding Author.
}

\begin{abstract}
Escalator is an essential large-scale public transportation equipment. Once the failure occurs, it will inevitably affect the operation and even cause safety accidents. As an important part of the structure of escalator, the loosening of the anchor bolt will lead to abnormal operation of escalator. Aiming at the current difficulty in extracting the fault features of anchor bolt loosening, a fault feature extraction method of escalator anchor loosening is constructed based on empirical wavelet transform (EWT) and bispectrum analysis. First, perform EWT decomposition of the original footing vibration acceleration signal to obtain a series of empirical mode functions (EMFs). Then, for each empirical mode function, the bispectrum was calculated by using bispectrum analysis method, and six texture features of the bispectrum were extracted as fault feature vectors by means of gray-gradient co-occurrence matrix. Finally, the extracted multi-scale fault feature vectors and bi-directional long short-term memory (BI-LSTM) were used to classify and identify the four types of fault signals with different degrees of foot loosening, and the fault types of foot loosening were determined. The results show that the feature extraction method based on empirical wavelet decomposition and bispectrum analysis can more effectively identify the loosening level of anchor bolts.
\end{abstract}

Keywords: Empirical wavelet decomposition, bispectrum analysis, fault identification, feature extraction, escalator

\section{Introduction}

Escalators have become an important transportation tool in modern city life. Escalators are commonly used in large shopping malls, railway stations and subway stations and other large buildings, transporting a large number of people and equipment every day. And the safety of escalators is closely related to the safety of the public. Once an escalator malfunction occurs, it may affect the operation in light cases or cause serious accidents, which can cause great damage to people's life and economy [1,2]. Due to frequent overtime and overload operation, the loosening of the anchor bolts of the escalator's mainframe footing occurs from time to time. As the key part of the escalator, the loosening of the fixing bolts of the mainframe feet will lead to periodic shocks during the operation of the escalator, which will cause the vibration of the escalator coupling system to intensify and affect the stability of the escalator operation, and will endanger the operation safety of the escalator in serious cases [3]. Therefore, condition monitoring and fault diagnosis of escalator bracket fixing bolts is essential.

The machine feet vibration signal of escalator is usually nonlinear and non-stationary $[4,5]$.In view of the nonlinear and non-stationary nature of mechanical fault signals and the characteristics of weak early fault characteristics and susceptibility to noise interference, numerous vibration signal fault diagnosis methods are proposed, such as short-time Fourier transform (STFT), wavelet transform (WT), empirical mode decomposition (EMD), variational mode decomposition (VMD), etc. However, the window function of STFT cannot be adaptively adjusted according to the frequency of the signal itself, which affects the accuracy of fault diagnosis [6, 7]. Selection of wavelet basis functions and decomposition layers of WT lacks adaptivity [8, 9]. Empirical mode decomposition (EMD) [10] has made a significant breakthrough in vibration signal fault information extraction, but EMD suffers from serious mode aliasing phenomena and endpoint effects, and lacks the necessary theoretical foundation [11,12]. In order to improve the shortcomings of EMD methods, many improved EMD algorithms have

ISSN: 0010-8189

C CONVERTER 2020

www.converter-magazine.info 
been proposed, such as local mean decomposition (LMD) [13], local characteristic-scale decomposition (LCD) [14], ensemble EMD (EEMD) [15], etc. However, these methods do not completely solve the drawbacks of EMD either. Variational mode decomposition (VMD) [16] overcomes the deficiencies of EMD and LMD. The signal decomposition is transformed into a variational problem to determine the center frequency and bandwidth of the component signals by seeking the optimal solution of the variational problem, so as to achieve the effective separation of the component signals. VMD has a sound theoretical basis and can better suppress modal aliasing. However, the combination of parameters and the number of decompositions of the penalty factor need to be determined before decomposition, because different combinations of parameters and the number of decompositions can affect the decomposition accuracy of the signal, which poses great difficulties to the accurate decomposition of the signal $[17,18]$

In recent years, Gilles [19] proposed the empirical wavelet transform (EWT) to perform adaptive decomposition of non-stationary signals. The main idea is to extract each mode component of the signal by constructing a series of suitable band-pass filter banks. When compared to EMD and VMD methods, EWT has a reliable mathematical theoretical basis and avoids modal aliasing and endpoint effects. Because EWT does not use iterative decomposition, it has less calculation and faster decomposition speed. Due to the above features, EWT has been widely used in the identification of fault information of rolling bearings [20, 21], fan bearings and fans [22]. For better fault identification for loosening of anchor bolt of escalator, this paper introduces EWT into the processing of vibration acceleration signals of escalator footing and uses EWT to perform mode decomposition of machine feet vibration signal. After decomposing the machine feet vibration signal into a group empirical mode function (EMF) by EWT, how to accurately extract the fault features from each mode component is another key issue in the identification of bolt loosening fault. The EMFs decomposed by EWT contain rich feature information, and different features represent different physical meanings. Choosing a suitable feature extraction method can significantly improve the recognition accuracy. Bispectrum analysis is a very effective algorithm, which contains all the phase information of the processed signal and can completely suppress the effect of Gaussian noise [23].Liu et al [24] applied it to the detection of microcracks under mixed frequency excitation. Wang et al [25] used Fractional bispectrum analysis to identify Fault Characteristic. And the results showed that for small cracks bispectrum analysis can effectively extract the fault features. Xu et al. [26] applied bispectrum analysis method to identify and analyze the signals of bearing. The experimental results verify the effectiveness of the bispectrum algorithm in microcrack feature extraction.

Based on EWT and bispectrum analysis, we proposed an fault identification method for loosening of anchor bolt of escalator, and used the proposed method to identify the degree of looseness. First, a set of empirical mode function (EMF) is obtained by multi-scale analysis of the machine feet vibration signal using empirical wavelet transform. Then, for each EMF, its bispectrum diagram and grey-gradient co-generation matrix are calculated using bispectrum analysis. And six fault features such as small-gradient dominance and grey-gradient average are extracted through the grey-gradient co-generation matrix. Finally, the fault eigenvalues of all mode components are combined into 18 dimensional fault eigenvectors, and Bidirectional long short-term memory (Bi-LSTM) is used to identify the loosening states of anchor bolt of escalator. The experimental analysis using the measured machine feet vibration signal shows that the proposed method can effectively identify the footing bolt loosening fault and determine the degree of bolt loosening.

\section{Theories of EWT and Bi-LSTM}

\section{$2.1 \mathrm{EWT}$}

Empirical wavelet transform (EWT) is constructed on basis of wavelet theory. EWT consists of two important steps: first, adaptive partitioning of the signal spectrum; then, the signal is decomposed using an orthogonal wavelet filter bank to obtain a modal component signal with tight support characteristics. Assum that the spectrum range of vibration signal after Fourier transform is $[0, \pi]$. Dividing the whole spectrum into $N$ segments, each 
segment of spectrum is denoted as $\Lambda_{n}=\left[\omega_{n-1}, \omega_{n}\right] n=1,2, \cdots, N . \omega_{0}$ and $\omega_{N}$ are the left and right boundaries of spectrum division respectively. Then the whole spectrum of signal can be represented as $\bigcup_{n=1}^{N} \Lambda_{n}=[0, \pi]$. EWT is a band-pass filter defined on each spectrum $\Lambda_{n}$. According to theory of the wavelet, the scaling function $\varphi(x)$ and wavelet function $\psi(x)$ of EWT are defined in frequency domain as follows ${ }^{[19]}$ :

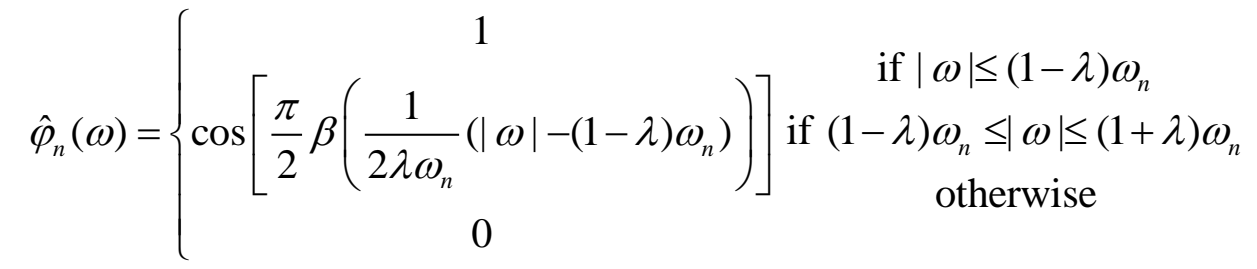

$$
\begin{aligned}
& \hat{\psi}_{n}(\omega)=\left\{\begin{array}{cc}
1 & \begin{array}{cc}
\cos \left[\frac{\pi}{2} \beta\left(\frac{1}{2 \lambda \omega_{n+1}}\left(|\omega|-(1-\lambda) \omega_{n+1}\right)\right)\right] & \begin{array}{c}
\text { if }(1+\lambda) \omega_{n} \leq|\omega| \leq(1-\lambda) \omega_{n+1} \\
\text { if }(1-\lambda) \omega_{n+1} \leq|\omega| \leq(1+\lambda) \omega_{n+1}
\end{array} \\
\sin \left[\frac{\pi}{2} \beta\left(\frac{1}{2 \lambda \omega_{n}}\left(|\omega|-(1-\lambda) \omega_{n}\right)\right)\right] & \text { if }(1-\lambda) \omega_{n} \leq|\omega| \leq(1+\lambda) \omega_{n} \\
0 & \text { otherwise }
\end{array}
\end{array}\right.
\end{aligned}
$$

where, $\beta_{n}=y^{4}\left(35-84 y+70 x^{2}-20 y^{3}\right), 0<\lambda<1$ and $\lambda<\min _{n}\left(\frac{\omega_{n+1}-\omega_{n}}{\omega_{n+1}+\omega_{n}}\right)$.

The decomposition of EWT is similar to that of classical wavelets. The decomposed detail and approximation coefficients are

$$
\begin{aligned}
& W_{g}^{\varepsilon}(n, x)=<g(x), \psi_{n}(x)>=\int g(\tau) \psi_{n}(\tau-x) d \tau=F^{-1}\left[\hat{g}(\omega) \hat{\psi}_{n}(\omega)\right] \\
& W_{g}^{\varepsilon}(0, x)=<g(x), \phi_{1}(x)>=\int g(\tau) \phi_{1}(\tau-x) d \tau=F^{-1}\left[\hat{g}(\omega) \hat{\phi}_{1}(\omega)\right]
\end{aligned}
$$

Where $F^{-1}(\cdot)$ denotes the inverse Fourier transform, and $\hat{\phi}_{1}(\omega)$ and $\hat{\psi}_{n}(\omega)$ are obtained by equations (1) and (2).

In the empirical wavelet transform, the reconstructing formula is

$$
g(x)=W_{g}^{\varepsilon}(0, x) \phi_{1}(x)+\sum_{n=1}^{N} W_{g}^{\varepsilon}(n, x) \psi_{n}(x)=F^{-1}\left[\hat{W}_{g}^{\varepsilon}(0, \omega) \hat{\phi}_{1}(\omega)+\sum_{n=1}^{N} \hat{W}_{f}^{\varepsilon}(n, \omega) \hat{\psi}_{n}(\omega)\right]
$$

According to formulas (3) and (4), the empirical mode function (EMF) formula after EWT decomposition can be calculated by the following formulas

$$
\left\{\begin{array}{c}
g_{0}(x)=W_{g}^{\varepsilon}(0, x) \phi_{1}(t) \\
g_{n}(x)=W_{g}^{\varepsilon}(n, x) \psi_{n}(x) \quad n=1,2, \cdots, N
\end{array}\right.
$$

A series of empirical mode functions (EMFs) can be obtained by machine feet vibration signal using EWT. ISSN: 0010-8189 
Bispectrum analysis is performed for each EMF to extract the fault eigenvectors for each mode.

\subsection{Bi-LSTM}

\subsubsection{LSTM}

Long short-term memory (LSTM) is an improved model based on recurrent neural network (RNN). The structure of LSTM is given in Fig. 1, which contains four main gate structures: input gate $i$, forget gate $f$, control gate $c$ and output gate $O$.

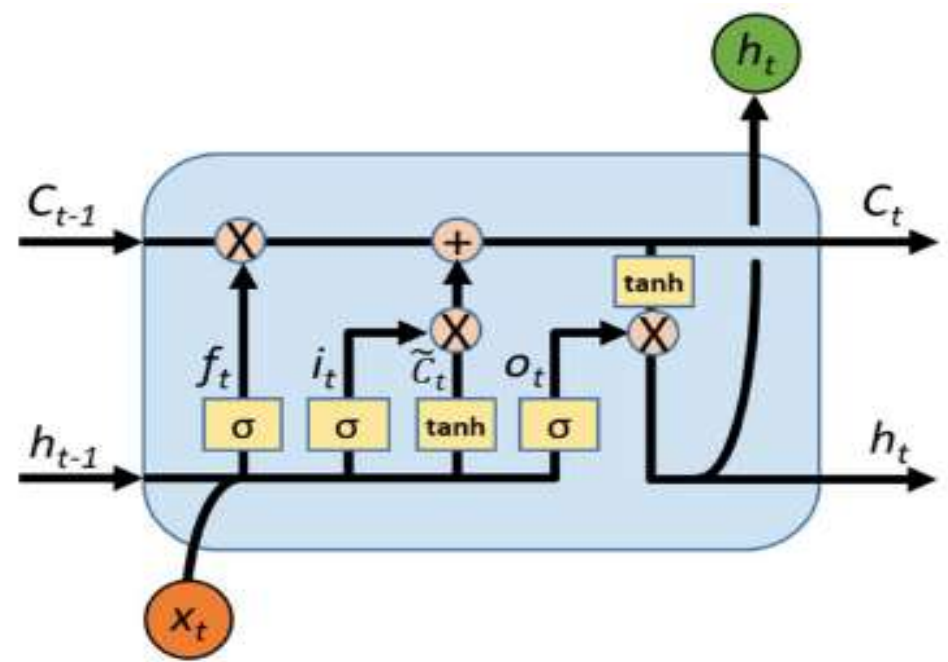

Fig 1: LSTM network structure diagram

The input gate $i$ determines which new information will be stored in the new cell state, and the moment $t$ input gate calculation is defined as

$$
\left.i_{t}=\sigma\left(W_{i} \llbracket h_{t-1}, x_{t}\right]+b_{i}\right)
$$

The forget gate $f$ determines which information should be ignored from prior memory, and the moment $t$ forgetting gate is calculated as defined as

$$
f_{t}=\sigma\left(W_{f}\left[h_{t-1}, x_{t}\right]+b_{f}\right)
$$

The control gate update the control unit status from $c_{t-1}$ to $c_{t}$ according to Eq. (6) and (7),

$$
\begin{aligned}
& \tilde{c}_{t}=\tanh \left(W_{c}\left[h_{t-1}, x_{t}\right]+b_{c}\right) \\
& c_{t}=f_{t} \circ c_{t-1}+i_{t} \circ \tilde{c}_{t}
\end{aligned}
$$

The output gate $O$ generats output and updates hidden vector $h_{t-1}$. The control process of output gate is defined as:

$$
\begin{aligned}
& o_{t}=\sigma\left(W_{o} \cdot\left[h_{t-1}, x_{t}\right]+b_{o}\right) \\
& h_{t}=o_{t} \circ \tanh \left(c_{t}\right)
\end{aligned}
$$


In Eq. (6)-(11): $h_{t}$ is the final output of the network; $\tilde{c}_{t}$ is the current input cell state; $c_{t}$ is the cell state at the current moment; $W_{i}, W_{f}, W_{c}$ and $W_{o}$ are the weight matrices of the four gated states respectively; $b_{i}, b_{f}, b_{c}$ and $b_{o}$ are the bias of each gated state respectively; $\sigma(\cdot)$ and $\tanh (\cdot)$ are the transfer functions; $\sqcup$ represents the vector inner product and the symbol $\circ$ denotes multiplication by elements.

\subsubsection{Bi-LSTM}

Bidirectional recurrent neural networks (BRNN) form a bidirectional network structure by adding a backpropagation layer to a recurrent neural network in order to use contextual information simultaneously. In the bidirectional network structure, the RNN units are replaced by LSTM units to form a bidirectional LSTM(BiLSTM).The structure of Bi-LSTM network is given in Fig.2, in which two independent LSTM networks are included to propagate information forward and backward respectively. Bi-LSTM network has the advantages of both RNN network and LSTM network. Bi-LSTM overcomes the decline problem of RNN network. Through parallel forward propagation network and backward propagation network, it can effectively estimate the impact of forward and backward events on current events.

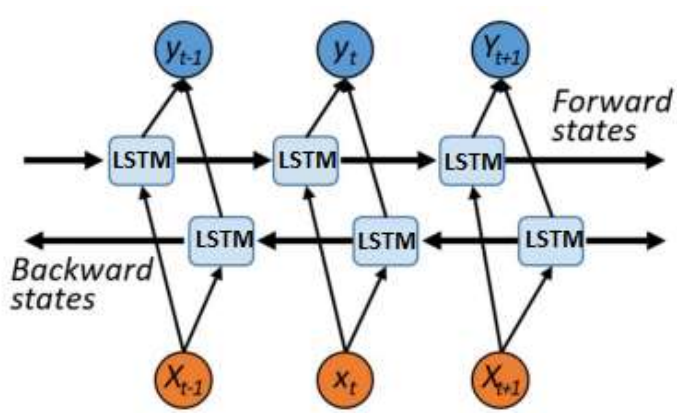

Fig 2: Bi-LSTM network structure diagram

\section{Multi scale fault feature extraction based on bispectrum analysis}

\subsection{Bispectrum analysis theory}

Bispectrum can effectively suppress Gaussian noise with high resolution, and can obtain the signal amplitude, phase, energy and other related information. Bispectrum analysis is simple to calculate, but still contains all the feature information of the higher-order spectrum. Therefore, in this paper, bispectrum analysis is used to extract information about the fault characteristics in the machine feet vibration signal. The steps of bispectrum calculation by direct method are as follows [23, 24]:

(a) The vibration signal to be analyzed is divided into $K$ segments, each segment containing $M$ samples. So the signal after segmentation is

$$
\boldsymbol{x}^{(k)}(n)=\left\{x^{(k)}(0), x^{(k)}(1), \cdots, x^{(k)}(M-1)\right\} \quad k=1,2, \cdots, K
$$

(b) For the $k$ th segment of data $\boldsymbol{x}^{(k)}(n)$, calculate its discrete Fourier transform.

$$
X^{(k)}(\lambda)=\frac{1}{M} \sum_{n=0}^{M-1} x^{(k)}(n) \exp \left(-j \frac{2 \pi n \lambda}{M}\right), \lambda=0,1, \cdots, M / 2 ; k=1,2, \cdots, K
$$

(c) Calculate the third-order autocorrelation coefficients of DFT.

ISSN: 0010-8189

(C) CONVERTER 2020

Www.converter-magazine.info 


$$
b_{k}\left(\lambda_{1}, \lambda_{2}\right)=\frac{1}{\Delta_{0}^{2}} \sum_{i_{1}=-L_{1}}^{L_{1}} \sum_{i_{2}=-L_{1}}^{L_{1}} X^{(k)}\left(\lambda_{1}+i_{1}\right) \cdot X^{(k)}\left(\lambda_{2}+i_{2}\right) \cdot X^{(k)}\left(-\lambda_{1}-\lambda_{2}-i_{1}-i_{2}\right)
$$

Where $\Delta_{0}=f_{s} / N_{0}, M=\left(2 L_{1}+1\right) N_{0}$. And $f_{s}$ denotes the sampling frequency.

$$
\text { Calculate the bispectrum estimation of vibration signal }
$$

$$
B\left(\omega_{1}, \omega_{2}\right)=\frac{1}{K} \sum_{k=1}^{K} b_{k}\left(\omega_{1}, \omega_{2}\right), \text { where } \omega_{1}=\frac{2 \pi f_{s}}{N_{0}} \lambda_{1}, \omega_{2}=\frac{2 \pi f_{s}}{N_{0}} \lambda_{2}
$$

It can be seen from the definition that bispectrum is a complex spectrum with two frequency variables $\omega_{1}$ and $\omega_{2}$. Bispectrum has 12 symmetrical regions in the frequency plane composed of $\omega_{1}$ and $\omega_{2}$. Only the bispectrum values in the main region need to be calculated, and then all bispectrum values in the $\left(\omega_{1}, \omega_{2}\right)$ plane can be calculated according to its symmetry.

\subsection{Bispectrum analysis of EWT for machine feet vibration signal}

For the complexity of the environment, the actual vibration signal collected from the footings of the escalator usually contains interfering noise. Bispectrum analysis can only effectively suppress Gaussian noise, but is powerless against non-Gaussian noise. Therefore, EWT is used to remove the effect of non-Gaussian noise from the signal before performing bispectrum analysis. After decomposition of the machine feet vibration signal using EWT, a series of EMFs are obtained. Because of the high noise frequency, the noise containing signal is often concentrated in the highest frequency modal component EMF1 after EWT decomposition ${ }^{[20,21]}$, while the fault feature information is contained in the remaining low frequency modal components. So this paper discards the first layer of empirical mode function EMF1 and only performs bispectrum analysis on the remaining mode functions to extract the fault feature information hidden in the mode components.

The sampling frequency of vibration signal of the escalator base foot is $2000 \mathrm{~Hz}$. The signals of normal, loosening 1 lap, loosening 2 laps and loosening 3 laps of fixed bolts are collected respectively, and some of the collected signals are intercepted as shown in Fig. 3. EWT decomposition was carried out for each of the four signals. And the number of frequency band intervals for EWT decomposition was taken as 4. The decomposed EMFs of the machine feet vibration signal with loosening 1 lap are shown in Fig. 4 (the EMFs of vibration signal of other cases are similar to this. Due to the limitation of space, they are not shown one by one). 


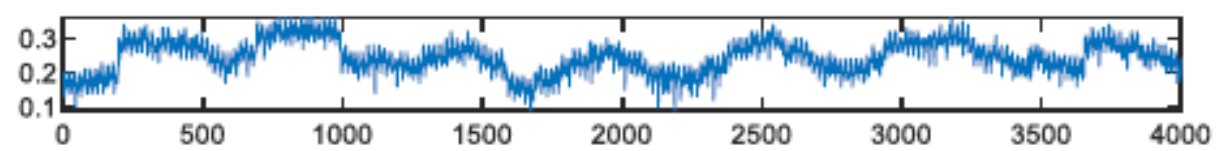

(a) Normal state vibration signal

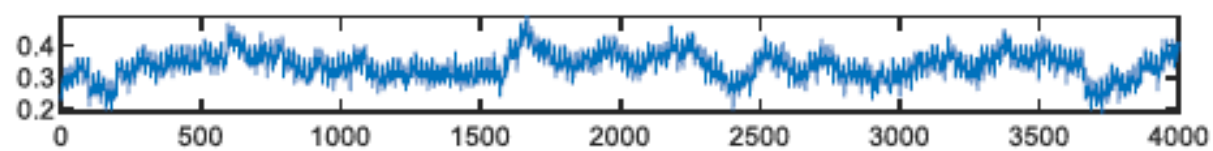

(b) Vibration signal when loosening one circle

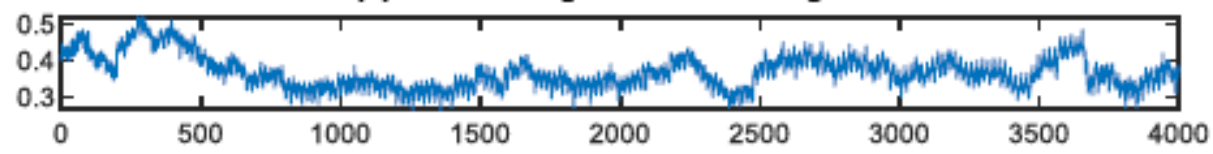

(c) Vibration signal when loosening two circle

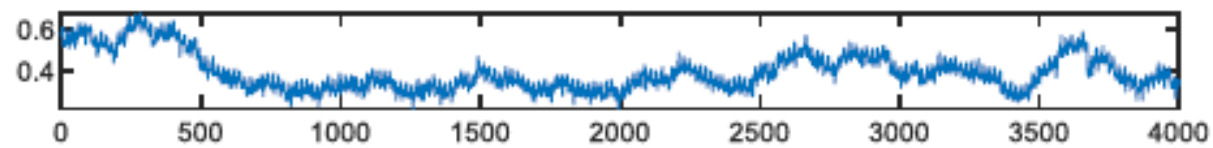

(d) Vibration signal when loosening four circle

Fig 3: The original machine feet vibration signal
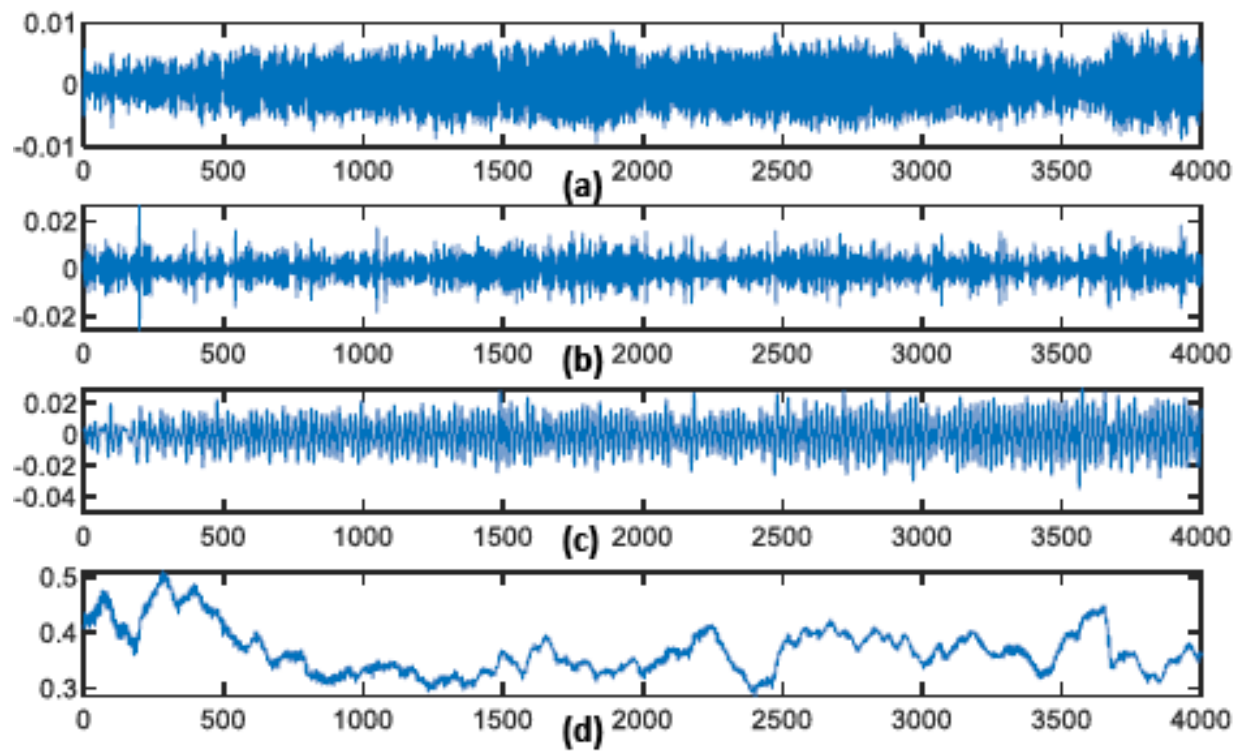

Fig 4: results of EWT decomposition (Loose two laps) (a) EMF1 (b) EMF2 (c) EMF3 (d) EMF4

The bispectrum analysis of mode function 2 (EMF2), mode function 3 (EMF3) and mode function 4 (EMF4) of four kinds of signals are carried out respectively. Fig. 5 is the two-dimensional contour plot of bispectrum analysis of machine feet vibration signal when the fixed bolt is normal. Fig. 6, Fig. 7 and Fig. 8 are the two-dimensional contour plot of bispectrum analysis of footing vibration signal when the fixed bolt is loosening for 1, 2 and 3 laps respectively.

ISSN: 0010-8189 

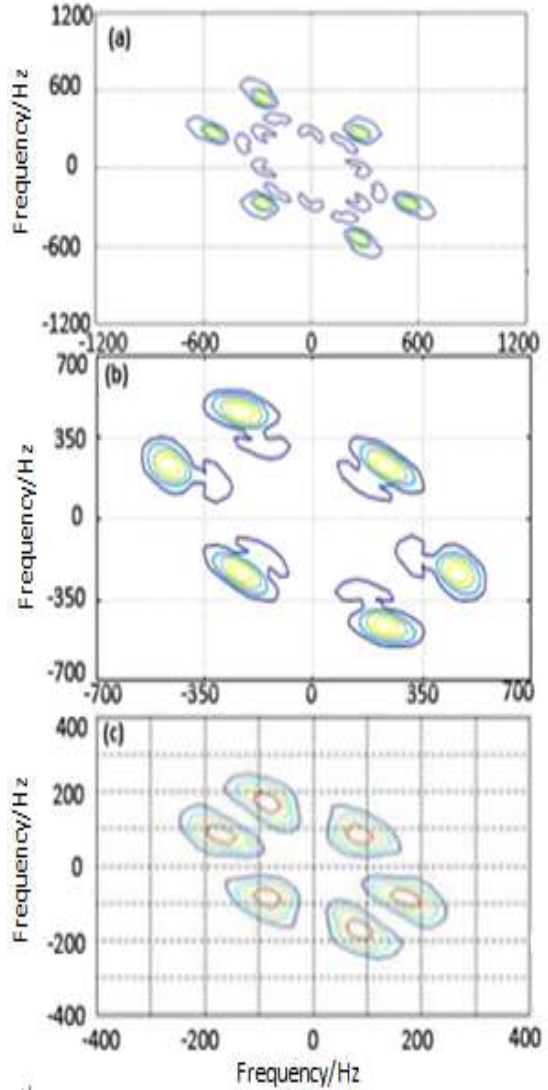

Fig 5: Contour plot of normal state (a) EMF2 (b) EMF3 (c) EMF4
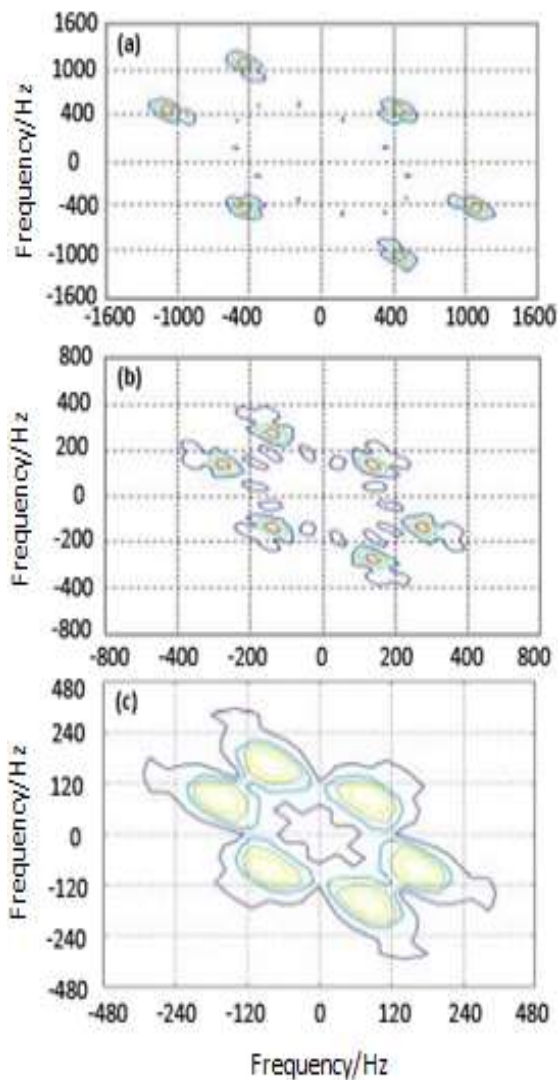

ISSN: 0010-8189Fig 7: Contour plot of loosening 2 laps C CONVERTER 202( (a) EMF2 (b) EMF3 (c) EMF4 www.converter-magazine.info
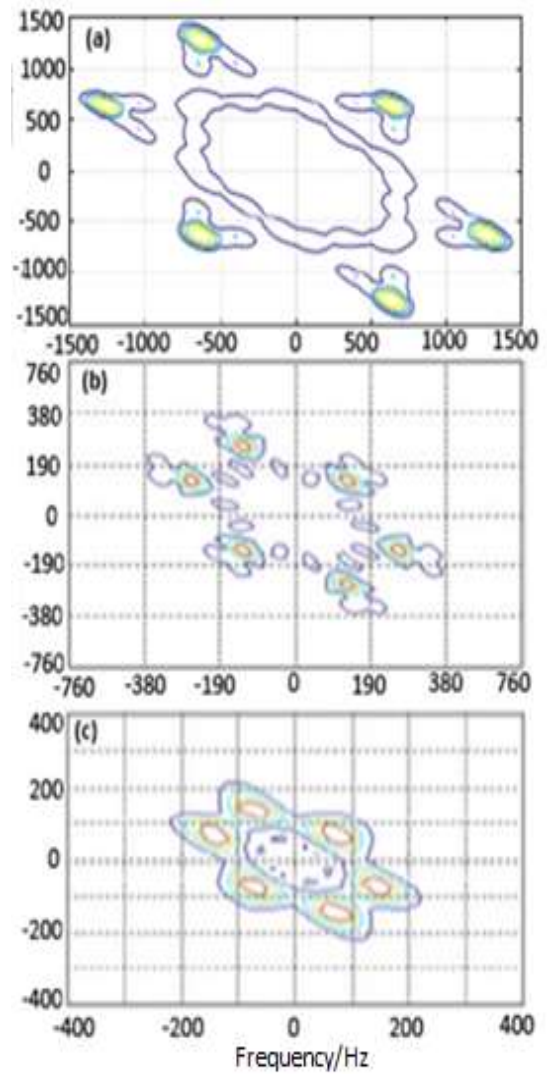

Fig 6: Contour plot of loosening 1 laps (a) EMF2 (b) EMF3 (c) EMF4
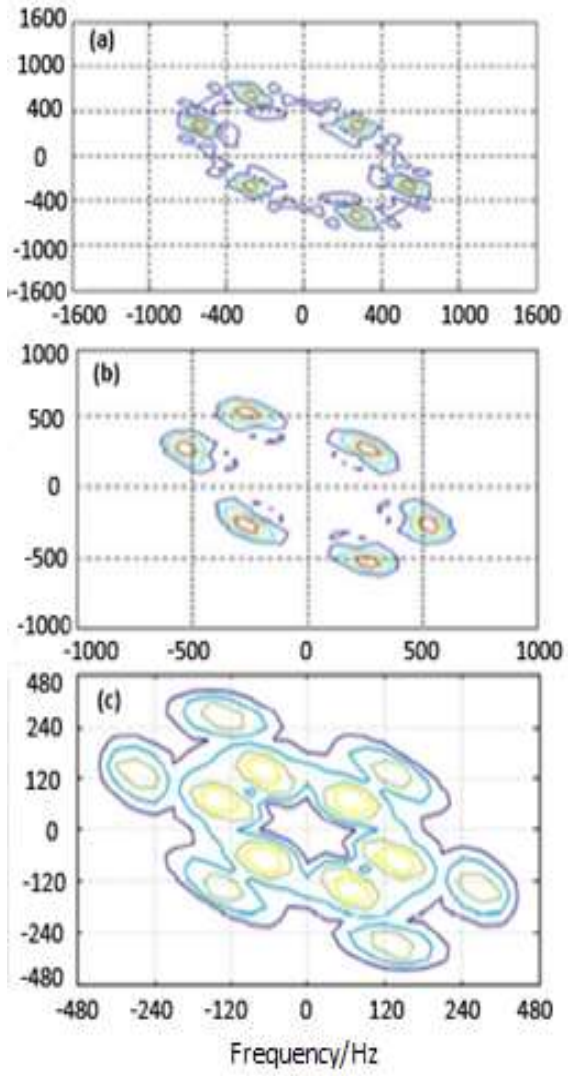

Fig 8: Contour plot of loosening 3 laps (a) EMF2 (b) EMF3 (c) EMF4 
From Fig. 5 to Fig. 8, this paper know that the two-dimensional spectrum of the escalator footing bolt when it is normal is obviously different from that when it is loosening. When the bolt is normal, the frequency converges to the center. While when the bolt is loose, the frequency expands outward gradually, and the frequency peak value also increases gradually. At the same time, the amplitude of bispectrum is different in different degrees of loosening.

\subsection{Fault feature extraction based on gray-gradient co-occurrence matrix}

From the two-dimensional contour plot of the bispectrum analysis of the machine feet vibration signal of escalator, it can be seen that there is a significant difference between the bispectrum information when the fixed bolt is normal and when the fixed bolt is loose. In order to automatically identify whether the bolt is loose and the degree of loosening, the feature information contained in the bispectrum coefficients needs to be extracted. The twodimensional contour plot of bispectrum analysis contains the basic feature information of the vibration signal, while its gradient plot depicts the edge and abrupt change information of the contour map. If both the twodimensional contour plot and its gradient plot are combined for feature extraction, more accurate fault characteristics can be obtained. Based on the fact that the grey-gradient co-occurrence matrix (GGCM) can describe both the gray information and the gradient information in the image, this paper constructs a grey-gradient co-occurrence matrix for the two-dimensional contour plot of the bispectrum analysis of vibration signals. The GGCM is used to extract the characteristic parameters of the bispectrum analysis contour map as the fault characteristics when the bolt is loose.

3.3.1 Normalization of gray matrix and gradient matrix

After gray processing of bispectrum analysis two-dimensional contour plot, gray matrix $f(m, n)$ is obtained. Because the pixel values of gray matrix are between $[0,255]$, there is no need for normalization. In this paper, only the gradient matrix of two-dimensional contour plot is normalized. Let $g(m, n)$ be the pixel value of point $(m, n)$ in the gradient matrix.

$$
g(m, n)=\sqrt{g_{x}^{2}(m, n)+g_{y}^{2}(m, n)}
$$

where,

$$
\begin{aligned}
& g_{x}(m, n)=f(m+1, n-1)+2 f(m+1, n)+f(m+1, n+1)-f(m-1, n-1)-2 f(m-1, n)-f(m-1, n+1) \\
& g_{y}(m, n)=f(m-1, n+1)+2 f(m, n+1)+f(m+1, n+1)-f(m-1, n+1)-2 f(m, n-1)-f(m+1, n-1) \\
& m=1,2, \cdots, M, \quad n=1,2, \cdots, N, M, N \text { represent the number of rows and columns of the gray matrix. }
\end{aligned}
$$

Let $g_{\max }$ be the maximum value in the gradient image and $L_{g}$ be the expected maximum gradient value after normalization. Then the normalized gradient matrix is

$$
G(m, n)=I N T\left[g(m, n) \cdot L_{g} / g_{\max }\right]+1
$$

In this paper, $L_{g}=64$. After normalization, two normalization matrices are obtained: gray normalization matrix $F(m, n)=f(m, n)$ and gradient normalization matrix $G(m, n)$. 
The gray-gradient co-occurrence matrix based on bispectrum analysis is $C=c(x, y)$. Then its element $c(x, y)$ is defined as the total number of pairs of image points with pixel values $x$ in the normalized grey matrix $F(m, n)$ and pixel values $y$ in the normalized gradient matrix $G(m, n)$. That is, $c(x, y)$ is equal to the number of pairs of image points that make $x=F(m, n)$ and $y=G(m, n)$. In order to facilitate texture feature extraction of the GGCM, it is necessary to normalise it. Let the element value of the normalised GGCM be $\hat{c}(x, y)$, then we have

$$
\hat{c}(x, y)=\frac{c(x, y)}{\sum_{x=0}^{255} \sum_{y=0}^{63} c(x, y)}
$$

3.3.3 Texture feature extraction with gray-gradient co-occurrence matrix

More feature information can be extracted from the GGCM of bispectrum analysis [25]. But if too many feature parameters are selected, this can lead to excessive computational effort and affect the accuracy of fault identification. Therefore, in this paper, six of these feature values are selected as the fault feature vectors for bolt loosening, the equations for each feature are as follows

(1) Small-gradient dominance: $T_{1}=\sum_{x=0}^{255} \sum_{y=0}^{63} \frac{\hat{c}(x, y)}{y^{2}} / \sum_{x=0}^{255} \sum_{y=0}^{63} \hat{c}(x, y)$

(2) Inhomogeneity of the grey distribution: $T_{2}=\sum_{x=0}^{255}\left[\sum_{y=0}^{63} \hat{c}(x, y)\right]^{2} / \sum_{x=0}^{255} \sum_{y=0}^{63} \hat{c}(x, y)$

(3) Inhomogeneity of the gradient distribution: $T_{3}=\sum_{y=0}^{63}\left[\sum_{x=0}^{225} \hat{c}(x, y)\right]^{2} / \sum_{x=0}^{255} \sum_{y=0}^{63} \hat{c}(x, y)$

(4) Grey entropy: $T_{4}=-\left\{\sum_{x=0}^{255}\left[\sum_{y=0}^{63} \hat{c}(x, y)\right]^{2} \cdot \log \left[\sum_{y=0}^{63} \hat{c}(x, y)\right]\right\}$

(5) Gradient entropy: $T_{5}=-\left\{\sum_{y=0}^{63}\left[\sum_{x=0}^{255} \hat{c}(x, y)\right]^{2} \cdot \log \left[\sum_{y=0}^{63} \hat{c}(x, y)\right]\right\}$

(6) Mixed entropy: $\quad T_{6}=\sum_{x=0}^{255} \sum_{y=0}^{63} \hat{c}(x, y) \log [\hat{c}(x, y)]$

Randomly select the normal state, loosening 1 lap, loosening 2 laps and loosening 3 laps data, and calculate the normalized values of EMF2, EMF3 and EMF4 texture features after EWT decomposition. The results are shown in Table 1.

Table 1 Normalized values of the 6 texture features of EMFs

\begin{tabular}{|c|c|c|c|c|c|c|c|}
\hline \multicolumn{2}{|c|}{ Texture features } & $\mathrm{T}_{1}$ & $\mathrm{~T}_{2}$ & $\mathrm{~T}_{3}$ & $\mathrm{~T}_{4}$ & $\mathrm{~T}_{5}$ & $\mathrm{~T}_{6}$ \\
\hline \multirow{4}{*}{\begin{tabular}{c} 
Normal state \\
\cline { 2 - 8 }
\end{tabular}} & $\mathrm{EMF}_{2}$ & 0.9300 & 0.5323 & 0.5475 & 0.5877 & 0.6980 & 0.8543 \\
\cline { 2 - 8 } & $\mathrm{EMF}_{4}$ & 0.8131 & 0.4646 & 0.7053 & 0.5132 & 0.6135 & 0.8037 \\
\hline \multirow{3}{*}{$\begin{array}{c}\text { Loosing } \\
\text { circle }\end{array}$} & $\mathrm{EMF}_{2}$ & 0.8342 & 0.6451 & 0.6937 & 0.7673 & 0.7835 & 0.9084 \\
\cline { 2 - 8 } & $\mathrm{EMF}_{3}$ & 0.9290 & 0.6900 & 0.9012 & 0.6945 & 0.7202 & 0.8586 \\
\cline { 2 - 8 } & $\mathrm{EMF}_{4}$ & 0.8431 & 0.7770 & 0.6888 & 0.5236 & 0.6049 & 0.7110 \\
\hline Loosing & $\mathrm{EMF}_{2}$ & 0.7490 & 0.4819 & 0.7825 & 0.8571 & 0.8846 & 0.9513 \\
\hline
\end{tabular}

ISSN: 0010-8189

C CONVERTER 2020 


\begin{tabular}{|c|l|l|l|l|l|l|l|}
\hline \multirow{2}{*}{2 circle } & $\mathrm{EMF}_{3}$ & 0.9411 & 0.6189 & 0.9459 & 0.7716 & 0.8318 & 0.8853 \\
\cline { 2 - 8 } & $\mathrm{EMF}_{4}$ & 0.7919 & 0.7212 & 0.7333 & 0.7143 & 0.7392 & 0.7728 \\
\hline \multirow{3}{*}{$\begin{array}{l}\text { Loosing } \\
3 \text { circles }\end{array}$} & $\mathrm{EMF}_{2}$ & 0.6391 & 0.7210 & 0.8546 & 0.9091 & 0.9160 & 0.9736 \\
\cline { 2 - 8 } & $\mathrm{EMF}_{3}$ & 0.7510 & 0.7323 & 0.9826 & 0.8553 & 0.8826 & 0.9012 \\
\cline { 2 - 8 } & $\mathrm{EMF}_{4}$ & 0.6964 & 0.8730 & 0.8768 & 0.7791 & 0.7956 & 0.7922 \\
\hline
\end{tabular}

\section{Bolt loosening fault diagnosis model based on EWT and Bispectrum}

In this paper, bidirectional LSTM is used to do fault identification for loosening of anchor bolt of escalator. Based on EWT bispectrum analysis and Bi-LSTM, the process of anchor bolt loosening fault diagnosis is shown in Fig. 9. The specific steps of bolt loosening fault identification are as follows:

a) EWT was used to decompose each training sample into four levels. And the last three order empirical mode function $(\mathrm{EMF})$ was retained;

b) EWT was used to decompose each training sample into four levels. And the last three order empirical mode function $(\mathrm{EMF})$ was retained;

c) Bispectrum analysis is performed on the last three order $\mathrm{EMFs}\left(\mathrm{EMF}_{2}, \mathrm{EMF}_{3}, \mathrm{EMF}_{4}\right)$ of each training sample. The gray matrix and gradient matrix are constructed by the two-dimensional contour plot of bispectrum analysis. Then the GGCM is constructed;

d) Six texture features are extracted from the GGCM of $\mathrm{EMF}_{k}(k=2,3,4)$ to form the 18 dimensional fault feature vector of training samples.

e) The Bi-LSTM network is trained with 160 sets of training data. And anchor bolt loosening fault diagnosis model is established.

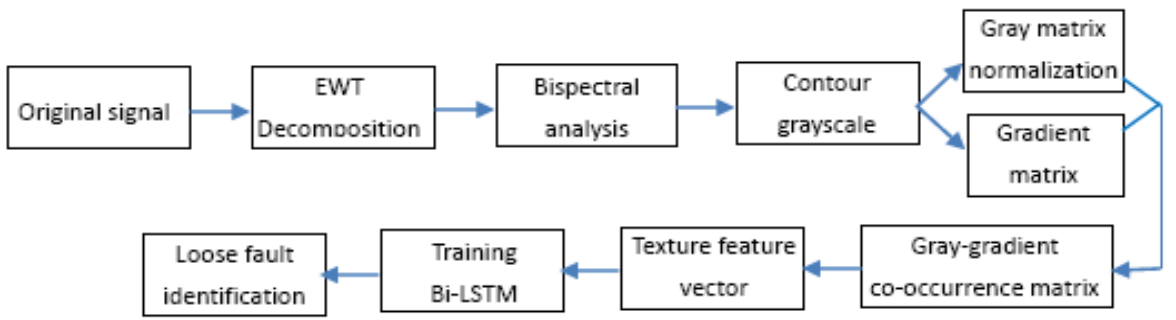

Fig 9: Process diagram of bolt loosening fault diagnosis

\section{Analysis of experimental results}

\subsection{Collection of experimental data}

This paper conducts simulation experiments on a Schindler S9700-30 escalator model with a vibration sensor sampling frequency of $2000 \mathrm{~Hz}$ and an acquisition time of $8 \mathrm{~s}$. The vibration sensor is installed on the anchor bolt position of the escalator through the thread. By adjusting the loosening degree of the anchor bolt, a total of 400 sets of experimental data were collected, including 100 groups of data when the anchor bolt was normal, 100 sets of data when the fixed bolt was loosening 1 lap, 100 sets of data when the fixed bolt was loosening 3 laps, and 100 groups of data when the fixed bolt was loosening 3 laps. According to the escalator product characteristics, the frequency range of the anchor bolt vibration is determined to be between $1 \sim 5 \mathrm{khz}$. So a low-pass filter is used to

ISSN: 0010-8189 
filter the high frequency part to avoid the interference signal being introduced into the next level. The filter circuit design uses an active filter circuit to filter out the spurious signal and then amplify the signal at the same time. During the collection process, a marker line is first drawn on the mainframe anchor bolt to record the initial position of the mainframe anchor bolt and to avoid excessive or inappropriate loosening of the mainframe anchor bolt. After drawing the marker line, the mainframe foot screw is loosening by 1,2 and 3 laps respectively against the marker line and confirmed by measurement that the mainframe anchor bolt is loosened in place.

\subsection{Identification results based on EWT-bispectrum analysis fault feature extraction}

EWT decomposition was carried out on the experimentally collected vibration data of the anchor bolt under normal and loosening conditions. EMF2, EMF3 and EMF4 were subjected to bispectrum analysis. And 6 texture features were extracted for each layer of EMF using a grey-gradient co-generation matrix to form an 18-dimensional fault feature vector, which is input into a Bi-LSTM network to identify the degree of loosening of the anchor bolt. The corresponding outputs are: "0" for a normal bolt, "1" for loosening 1 lap, "2" for loosening 2 laps and "3" for loosening 3 laps.In Bi-LSTM, the number of neurons in the input layer, output layer and hidden layer are 30,1 and 20 respectively. The internal parameters of the LSTM were trained using Adam's algorithm with a learning rate of 0.001 , a training number of 1000 , and a training target of 0.0001 . The parameters of the reverse layer network were the same as those of the forward layer.

The experimental results of the proposed method are compared with Bispectrum, EMD and bispectrum (EMDBispec) and ensemble EMD and bispectrum(EEMD-Bispec) for comparative analysis,. In the EMD-Bispec method, the first layer of IMF after EMD decomposition is discarded. And the IMF2-IMF4 are selected as the object of study. Bispectrum analysis and feature extraction are performed for each layer of IMF. And then the feature vectors are fed to the Bi-LSTM network for identification. In the EEMD-Bispec method, Gaussian white noise is added with an intensity of 0.5 and a number of 200 additions. The 2-4 layer IMFs after EEMD decomposition are taken as the object of study. The bispectrum analysis feature vectors of each layer IMF are calculated. And then the combination of each layer feature vector is inputted into the Bi-LSTM network for identification. A comparison of the correct identification rates of the test samples for the four methods is shown in Fig. 10, and the correct diagnostic results are shown in Table 2.

Table 2 Accuracy rate of fault identification for loosening of anchor bolt of escalator

\begin{tabular}{|l|c|c|c|c|c|c|c|c|}
\hline & \multicolumn{2}{|c|}{ Bi-Spectrum } & \multicolumn{2}{c|}{ EMD-Spectrum } & \multicolumn{2}{c|}{ EEMD-Spectrum } & \multicolumn{2}{c|}{ EWT-Spectrum } \\
\hline \multicolumn{1}{|c|}{ state } & $\begin{array}{c}\text { identified/ } \\
\text { samples }\end{array}$ & $\begin{array}{c}\text { Accuracy } \\
/ \%\end{array}$ & $\begin{array}{c}\text { identified/ } \\
\text { samples }\end{array}$ & $\begin{array}{c}\text { Accuracy } \\
/ \%\end{array}$ & $\begin{array}{c}\text { identified/ } \\
\text { samples }\end{array}$ & $\begin{array}{c}\text { Accuracy } \\
/ \%\end{array}$ & $\begin{array}{c}\text { identified/ } \\
\text { samples }\end{array}$ & $\begin{array}{c}\text { Accuracy } \\
/ \%\end{array}$ \\
\hline $\begin{array}{l}\text { Normal } \\
\text { state }\end{array}$ & $34 / 40$ & 85.0 & $36 / 40$ & 90.0 & $38 / 40$ & 95.0 & $40 / 40$ & 100 \\
\hline $\begin{array}{l}\text { Loosing } \\
1 \text { circle }\end{array}$ & $27 / 40$ & 67.5 & $33 / 40$ & 82.5 & $35 / 40$ & 87.5 & $37 / 40$ & 92.5 \\
\hline $\begin{array}{l}\text { Loosing } \\
2 \text { circle }\end{array}$ & $29 / 40$ & 72.5 & $34 / 40$ & 85.0 & $37 / 40$ & 92.5 & $38 / 40$ & 95.0 \\
\hline $\begin{array}{l}\text { Loosing } \\
3 \text { circle }\end{array}$ & $32 / 40$ & 80.0 & $34 / 40$ & 85.0 & $34 / 40$ & 85.0 & $38 / 40$ & 95.0 \\
\hline whole & $122 / 160$ & 76.25 & $137 / 160$ & 85.62 & $144 / 160$ & 90.0 & $152 / 160$ & 95.63 \\
\hline
\end{tabular}




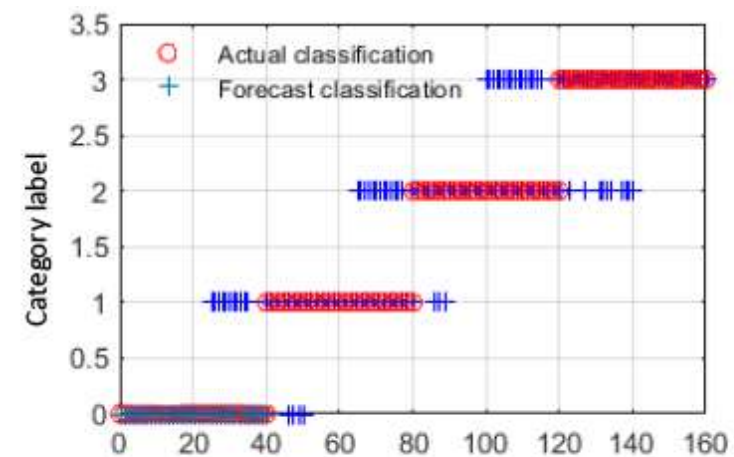

(a)

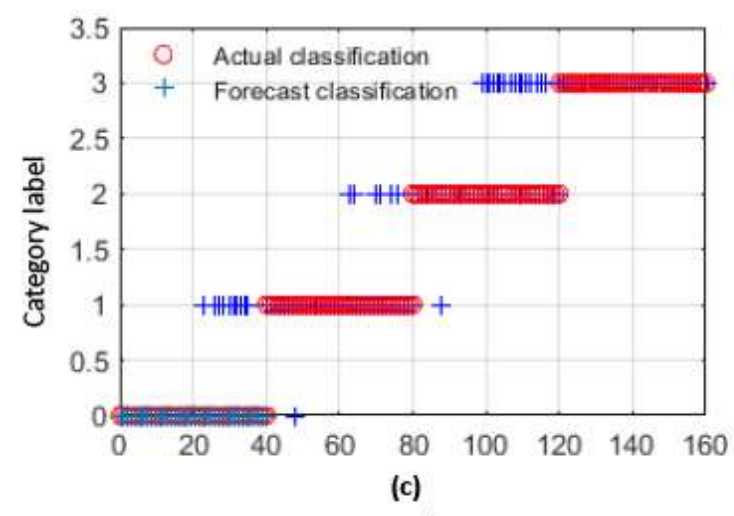

Test sample set

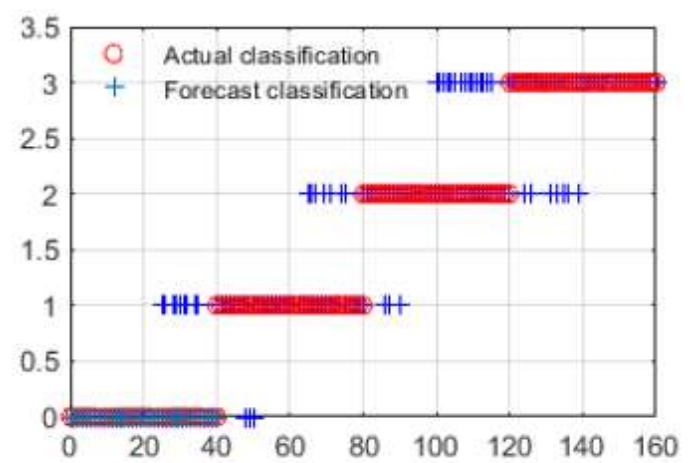

(b)

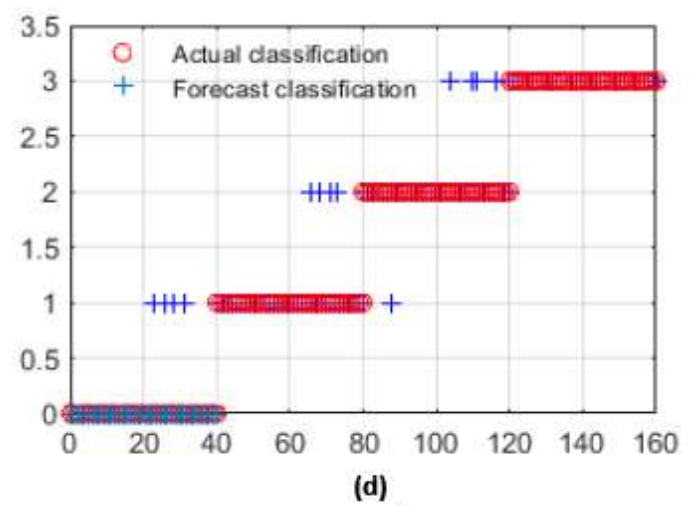

Test sample set

Fig 10: Diagnosis results of fixing bolt loosening degree (a) Bispectrum (b) EMD-Bispec (c) EEMD-Bispec (d) EWT-Bispec

From Fig. 10 and Table 2, this paper know that the bispectrum analysis method has the lowest recognition accuracy, with an overall recognition rate of only $76.25 \%$. This is because the bispectrum analysis method does not carry out multi-scale decomposition of the original vibration signal and extracts the fault features directly. But the components of different modes in the signal are mixed together. At the same time, the interference of non gaussian noise also has a certain impact on the feature extraction. The recognition accuracy of the EMD-Bispec method is significantly higher than that of the bispectrum analysis method, with an overall recognition rate of $85.62 \%$. This is because the EMD suppresses non-Gaussian noise to a certain extent and separates the mode components of the original vibration signal. Thus it allows more accurate extraction of fault features. Compared with EMD-Bispec method, the overall recognition rate of EEMD-Bispec method is improved to 90\%, mainly because EEMD improves the mode aliasing of EMD t. Compared with EEMD-Bispec method, the recognition accuracy of the proposed EWT-Bispec method proposed is improved. Because EEMD still has serious endpoint effect and a certain degree of mode aliasing, which affects the separation of different scale feature information. And the separated mode components still contain different scale vibration information. EWT uses band-pass filter banks to extract the mode aliasing, and avoids the modal aliasing and endpoint effect, which can separate the fault information of different scales as much as possible. Therefore, EWT decomposition can extract more accurate features of bolt loosening fault diagnosis through bispectrum analysis.

Overall, the method in this paper has improved the overall accuracy by about $21.38 \%$ compared to the Bispectrum method, about $10.01 \%$ compared to the EMD-Bispec method, and about $6.53 \%$ compared to the EEMD-Bispec method.

\section{Conclusion}

We proposed a new method based on empirical wavelet decomposition and bispectrum (EWT-Bispectrum) for ISSN: 0010-8189 
identificating the loosening degree of anchor bolt of escalator. The multi-scale decomposition of the machine feet vibration signal is carried out by using EWT. Then the fault features are extracted from the empirical mode functions (EMFs) of different scales by using the gray-gradient co-generation matrix after Bispectrum analysis. Establish a fault diagnosis model by using of Bi-LSTM. And then the feet vibration signal is diagnosed and analyzed based on this model to identify the degree of looseness of the fixed bolts. The experimental results indicate that the proposed method is able to identify the loosening faults of the footing bolts more accurately and achieve the accurate diagnosis of the loosening degree of the fixed bolts. Thus effectively preventing the occurrence of escalator footing loosening accidents. In the current study, after the vibration signal of the foundation is decomposed by EWT, all components are processed by bispectral analysis and fault features are extracted, which results in a large amount of calculation. However, the present studies have shown that the fault information is mainly concentrated in a few empirical mode function components (EMF) after the fault signal is decomposed by EWT. Therefore, how to use spectral kurtosis and other methods to select the EMF containing the main fault information to further improve the fault identification accuracy and reduce the computation, which is our next step.

\section{Acknowledgements}

This research was supported by National Natural Science Foundation of China (Grant No. 61671338).

\section{References}

[1] L.H. Liu, W.P. Long, "Study on the cause and prevention of subway escalator passenger injury accident," Chinese Railways, vol.5, no. 11, pp.2725-2746, 2011.

[2] F. Bonnardot, K. Lizoul, S. Errafik, H. Ander, F. Guillet, "High frequency demodulation technique for instantaneous angular speed estimation," Mechanical System and Signal Processing, vol.152, no.107745, 2021.

[3] W.X. Wang, Z.L. Yang, X.G. Hua, Z.Q. Chen, X.Y. Wang, G.B. Song, "Evaluation of a pendulum pounding tuned mass damper for seismic control of structures," Engineering Structures, vol.228,no. $111554,2021$.

[4] Q.Y. Meng, "Research on fault diagnosis method for main drive shaft bearing of escalator based EEMD-SVM,” Machinery \& Electronics, vol.38, no.5, pp.51-53, 2020.

[5] H.X. Ma, Z.X. Li, Z.D. Wang, R.J. Feng, G. Li, J.Y. Xu, "Research on measuring device and quantifiable risk assessment method based on FMEA of escalator brake," Advances in Mechanical Engineering, vol.13, no.3, pp.1-17, 2021.

[6] A. Karimpour, S. Rahmatalla, "Extended Empirical Wavelet Transformation: Application to structural updating,” Journal of Sound and Vibration, vol.500, no.116026, 2021.

[7] V. Sharma, N.K. Raghuwanshi, A.K. Jain, "Sensitive Sub-band Selection Criteria for Empirical Wavelet Transform to Detect Bearing Fault Based on Vibration Signals," Journal of Vibration Engineering \& Technologies, 2021.

[8] K.T. Chui, B.B. Gupta, R.W. Liu, P. Vasant, "Handling Data Heterogeneity in Electricity Load Disaggregation via Optimized Complete Ensemble Empirical Mode Decomposition and Wavelet Packet Transform," Sensors, vol.21, no.9, pp.3133, 2021.

[9] J.T. Li, J. Guo, X.Q. Zhu, "Time-Varying Parameter Identification of Bridges Subject to Moving Vehicles Using Ridge Extraction Based on Empirical Wavelet Transform," International Journal of Structural Stability and Dynamics, vol.21, no.4, pp.2150046, 2021.

[10] Y.J. Sun, S.H. Li, Y.L. Wang, X.H. Wang, "Fault diagnosis of rolling bearing based on empirical mode decomposition and improved manhattan distance in symmetrized dot pattern image,” Mechanical Systems and Signal Processing, vo.159, pp.107817, 2021.

[11] S. Wang, P.J. Niu, Q. H. Guo, X. C. Wang, F.Z. Wang, "An adaptive empirical mode decomposition and stochastic resonance system in high efficient detection of terahertz radar signal," Ferroelectrics, vo.563, no.1, pp.148-160, 2020.

ISSN: 0010-8189 
[12] J. M. Antelis, C.A. Rivera, E. Galvis, A. F. Ruiz-Olaya, "Detection of SSVEP based on empirical mode decomposition and power spectrum peaks analysis," Biocybernetics and Biomedical Engineering, vol.40, no.3, pp.1010-1021, 2020.

[13] T. Lu, F.Q.H. Yu, J.R. Wang, et al, "Application of adaptive complementary ensemble local mean decomposition in underwater acoustic signal processin," Applied Acoustics, vol.178, no.107966, 2021.

[14] W. Du, L.Q. Fang, "Fault diagnosis of automaton based on local characteristic-scale decomposition and individual feature selection," IOP Conference Series-Earth and Environmental Science, vol.69, pp.012076, 2017.

[15] R. N. Toma, C. H. Kim, J.M. Kim, "Bearing Fault Classification Using Ensemble Empirical Mode Decomposition and Convolutional Neural Network", Electronics, vol.10, no.11, pp.1248, 2021.

[16] H.L. Wang, F. Wu, L. Zhang, "Application of variational mode decomposition optimized with improved whale optimization algorithm in bearing failure diagnosis," Alexandria Engineering Journal, vol.60, no.5, pp.4689-4699, 2021.

[17] M. Chouksey, R.K. Jha, "A multiverse optimization based colour image segmentation using variational mode decomposition," Expert System with Applications, vol.171, pp.114587, 2021.

[18] X.A. Yan, Y. Liu, W. Zhang, M.P. Jia, X.B. Wang, "Research on a Novel Improved Adaptive Variational Mode Decomposition Method in Rotor Fault Diagnosis,” Applied Sciences-Basel, vol.10, no.5, pp.1696, 2020.

[19] H. Li, J. Lin, N.H. Liu, F.Y. Li, J H. Gao, "Seismic Reservoir Delineation via Hankel Transform Based Enhanced Empirical Wavelet Transform," IEEE Geoscience and Remote Sensing Letters, vol.17, no.8, pp.1411-1414, 2020.

[20] J.M. Ding, "A double impulsiveness measurement indices-bilaterally driven empirical wavelet transform and its application to wheelset-bearing-system compound fault detection," Measurement, vol.175, pp.109135, 2021.

[21] K. Zhang, C.Y. Ma, Y.G. Xu, P. Chen, J.X. Du, "Feature extraction method based on adaptive and concise empirical wavelet transform and its applications in bearing fault diagnosis," Measurement, vol.172, pp.108976, 2021.

[22] J.J. He, C. Song, Q.W. Luo, C.H. Yang, W.H. Gui, "WT-ASG: Empirical Wavelet Transform with Adaptive Savitzky-Golay Filtering for TDLAS,” IEEE Photonics Journal, vol.12, no.3, pp, 2020.

[23] I. Mitiche, M.D. Jenkins, P. Boreham, A. Nesbitt, G. Morison, “An expert system for EMI data classification based on complex Bispectrum representation and deep learning methods," Expert System with Applications, vol.171, pp.114568, 2021.

[24] H. Liu, Z.B. Wang, B. Zheng, "Surface crack characterization using laser nonlinear ultrasonics based on the bispectrum," Measurement Science and Technology, vol.32, no.3, pp.035204, 2021.

[25] H.B. Wang, J.B. Long, Z.L. Liu, F.You, "Fault Characteristic Extraction by Fractional Lower-Order Bispectrum Methods," Mathematical Problems in Engineering, vol.2020, pp.8823389, 2020.

[26] Y.D. Xu, C. Fu, N.Hu, B.S. Huang, F.S. Gu, A.D.Ball, “A phase linearisation-based modulation signal bispectrum for analysing cyclostationary bearing signals," Structural Health Monitoring-an Internaional Journal, vol.20, no.3, pp.1231-1246, 2020. 